\title{
Influence of fermentation activity of typical chernozem on content and hydrophobic-and-hydrophilic properties of humic acids at different techniques of soil cultivation
}

Skrylnyk Ye.,

Makliuk O.,

Popirniy M.

National Scientific Center "Institute for soil science and agrochemical researches named after $O$.

N. Sokolovskiy", Kharkiv

The purpose. To study influence of fermentation activity of oxide-reductases of typical chernozem on content and hydrophobic-and-hydrophilic properties of humic acids $(\mathrm{HA})$ at different techniques of soil cultivation. Methods. Field, laboratory-analytical, spectrometric. Results. Increase of polyphenoloxidase activity for 10-years use of zero cultivation promotes heightening of hydrophoby of HA due to formation of big quantity of condensed non-polar aromatic structures. After ploughing occurs re-organization of conformation of macrostructure of HA, which is caused by formation of more polar hydrophilous structures of HA in view of changing oxide-reductase activity, that in its turn causes greater moving ability of humus in comparison with zero cultivation and layland. Conclusions. Use of zero cultivation promotes reverse re-organization of functional macrostructure of HA due to change of oxide-reductase activity aside formation of hydrophobic structures - non-polar aromatic compounds.

Key words: oxide-reductase, soil cultivation, humic acids, non-polar aromatic structures, hydrophobicand-hydrophilic properties, re-organization of conformation.

Enzymatic activity of soil is an important factor forming quantitative and qualitative properties of humus. Changes in these properties occur after tillage of virgin black soil with different methods. These changes affect primarily the processes of biodegradation of fresh organic matter; secondly, the formation of structural elements of humic substances (HS), thereby causing changes in composition of soil organic matter (SOM) [1]. Transforming of the composition of humus causes macroscopic structural reorganization that defines functional properties of HS to the accumulation and migration of different elements in the ecosystem [2]. The macrostructural organization of HS is presented by supramolecular macrostructure complexes (supramolecular), which are formed as a result of self-assembling of different organic heterogeneous smaller molecules and their molecular associates, formed as a result of biodegradation of dead organic residues [3].

Self-assembling process is due to the thermodynamic most stable organic structures; the nature of aggregation depends on $\mathrm{pH}$ and causes hydrophobic and hydrophilic properties (amphiphile) of HS [3-5]. Stabilization of HS supramolecular conformation is due to various weak intermolecular interactions (hydrophobic, van der Waals, $\pi-\pi, \pi-\mathrm{CH}$ et al.). These complexes cause multifunctionality of HS. The strong covalent bonds form the most stable structural elements, namely unpolar aromatic structures that due to selfassembling and hydrophobic molecular $\pi-\pi$ association form stable hydrophobic domains $[4,5]$. It is known that the highest carbon content is in a covalently conjugated $(C=C)$ aromatic structures that are low molecular weight compounds [2], and their hydrophobic aggregation forms stable nonpolar molecular domains, but their hydrophilic aggregation forms polar mobile and active complexes such as fulvic acids (FA) $[3,4]$. The largest numbers of aromatic hydrophobic domains are in humic acids (HA), which provide high carbon content in typical black soil through active microbiological activity. It is shown that hydrophobic fused "core" of HA presented poorly conjugated fused aromatic and heterocyclic structures (2-3 rings such naphthalene, anthracene) different from highly condensed structure of coal [6] Formation of condensed aromatic structures occurs by free radical oxidation of quinones through semiquinone (SQ) radicals [2]. The 
process of condensation thanks to free radical reactions is catalyzed by polyphenoloxidase and leads to oxidase induced polymerization and formation of covalent connections (such as -C-O-, -C-C- bonds) between structure elements that form nonpolar aromatic structure [3]. Important structure elements of SOM are condensed structures with oxygen containing functional groups: ketones $(-\mathrm{C}=\mathrm{O})$ and carboxyl group ($\mathrm{COOH})$. Methoxyl groups $(-\mathrm{OCH} 3)$ of lignin SOM structures are subjected to dehydration processes, affecting the conformation arrangement and the activity of oxygen-containing functional groups [3 -5].

Intensive tillage of black soil causes the amplification process of mineralization of SOM. This leads to dehumification that cause irreversible reorganization of supramolecular complex of SOM followed by the deterioration of functional self-regulating humus macrostructure $[1,6]$. In this connection in the practice of agriculture was introduced tillage saving technologies that will contribute to restore biodiversity, soil fertility and improve the ecological state as a whole. [7] Among these technologies is "No tillage" system (No till) successfully used in various western countries to improve content quality humus and stabilize global $\mathrm{CO}_{2}$ [1]. Therefore, the study of changes in the composition of structural components in HA macrostructure and their amphiphile properties caused by the change of enzymatic processes for different soil conditions, is relevant and appropriate. The purpose of this study is to identify the impact of different tillage due to changes in oxidoreductase activity on the content of aromatic structures and amphiphile properties of HA.

Materials and methods. The samples of soil have been collected in the experimental field of the V.V. Dokuchayev Kharkiv National Agrarian University, the Department of Agriculture. The field experiment was started in 2006. The variants of the tillage investigated in the field experiment are as follows: 1) 40 year non-tilled typical black soil (control); 2) soil intensively tilled (traditional plowing depth of $20-22 \mathrm{~cm}$ ); 3) Nontilled soil. Soil samples have been collected from the surface soil layer of $0-20 \mathrm{~cm}$. Determination of oxidoreductase activity (soil dehydrogenase and polyphenoloxidaiy activity) was performed according to the method [8]. Extraction of HA from the soil samples was carried out by ISO 7606: 2014 [9].

The transmissions Fourier Infrared Spectroscopy (FT-IR) of the HA has been performed by the Agilent Technologies Cary Spectrometer 630 in the standard measurement area of $500-4000 \mathrm{~cm}^{-1}$ (in a potassium bromide compressed tablet). Analysis of the electron spin resonance (ESR) characteristics has been performed using spectrometer JES-ME-3x, which operates in the three-centimeter wavelength range (Xband) with a frequency modulation of $100 \mathrm{kHz}$. Samples of HA are placed in a quartz ampoule produced by JEOL Company. The sample of polycrystalline of diphenylpicrylhydrazyl (DPPH) has been used as a standard for assessment of the g-factors and intensities of the ESR spectra (Gauss); the average value of the $\mathrm{g}$-factor has equaled to $2.0036 \pm 0.0003$ that is similar with free electron. Size-exclusion chromatography (gel-filtration chromatography) was performed using Sephadex G-200 gel. Elution (output fractions) has been performed with a solution of $0.1 \mathrm{~N} \mathrm{NaOH}$. Registration of molecular fractions was performed by the absorption spectrum of aromatic structures and their functional groups at $465 \mathrm{~nm}$ wavelength.

Results of research. The activity of oxidoreductase of typical black soil decreases under intensive tillage, namely activity of polyphenoloxidase decreases after intensive tillage (plowing) as compared to control soil (Fig. 1). 


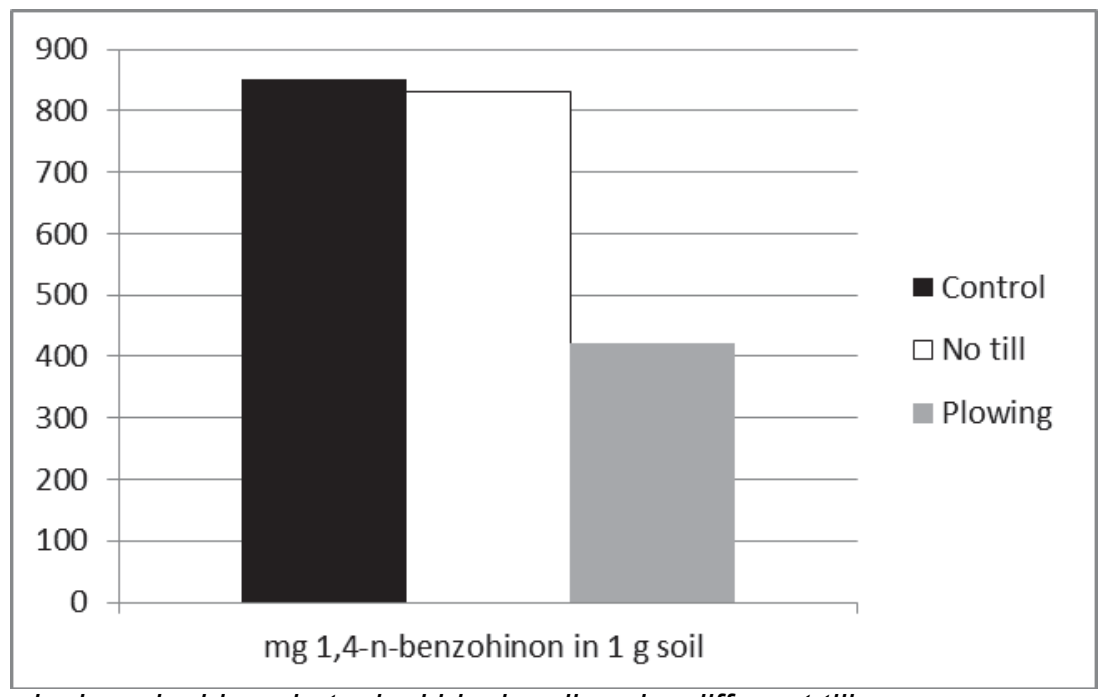

Fig. 1. Activity of polyphenoloxidase in typical black soil under different tillage

In previous work was revealed decrease of humus content due to enhancing of mineralization process after plowing in this experimental field [10]. Increase of activity of biodegradation in SOM, due to mixing of deeper layers of soil, reduces polyphenoloxidase activity, but increases activity of dehydrogenase (Fig. 2). The hydrogen is cleaved from the substrate due to deblocking of oxygen containing functional groups of HA thanks to better aeration of $0-20 \mathrm{~cm}$ layer of the soil.

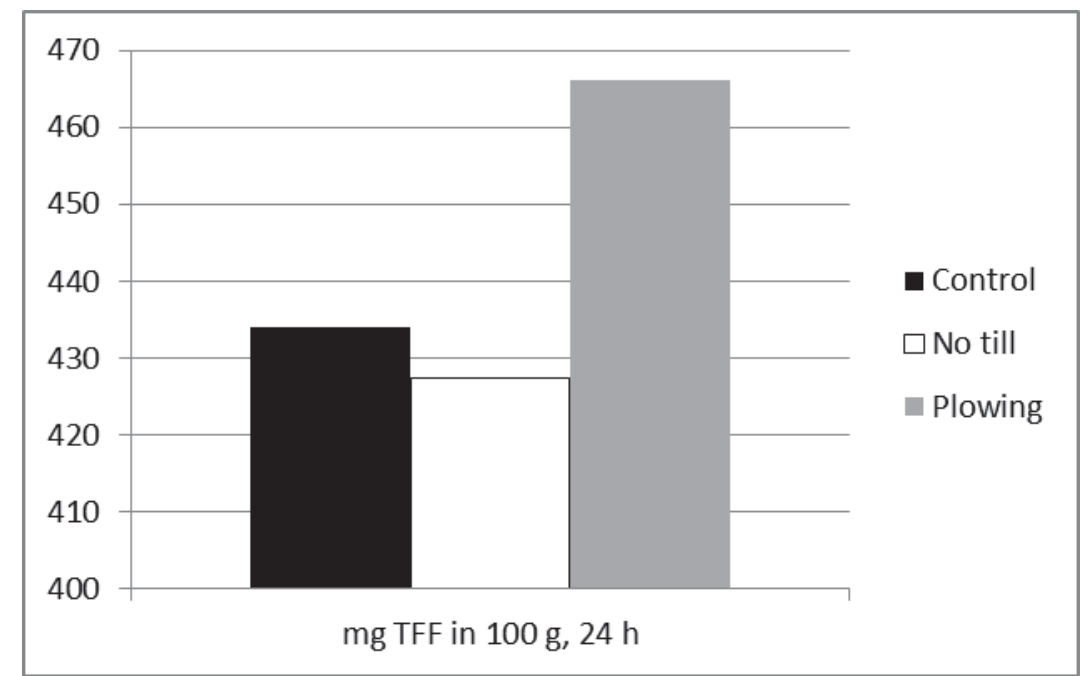

Fig. 1. Activity of dehydrogenase in typical black soil under different tillage

Increased activity of dehydrogenase is associated with biodegradation of fresh SOM and decrease of the ratio of carbon to nitrogen [10]. The plowing changes the direction of transformation of SOM, due to dehydration processes activation, and reduction of polyphenoloxidase activity that determines the decrease of stable compounds, and increasing the biological mineralization of less stable components of SOM.

All FT-IR spectra of HA samples (Fig. 3) clearly show structural elements of the HA, which differ in relative absorption amplitude. In the short-wave region the obtained spectra is presented by broad absorption band with a maximum of $3450 \mathrm{~cm}^{-1}$. Its presence is associated with hydroxyl and phenol hydroxyl groups $(\mathrm{OH})$ involved in intermolecular $\mathrm{H}$ - bonds. Doublet bands at $2900 \mathrm{~cm}^{-1}$ and $2800 \mathrm{~cm}^{-1}$, due stretching vibrations $\mathrm{C}-\mathrm{H}$ in $\mathrm{CH}_{3}$ and $\mathrm{CH}_{2}$ - groups and confirmed the presence of deformation bands of ionized methoxyl groups $1440 \mathrm{~cm}^{-1}$ and $1375 \mathrm{~cm}^{-1}$. Oxygen containing functional groups are shown by the 1710 $1725 \mathrm{~cm}^{-1}$ due to vibrations of $\mathrm{C}=\mathrm{O}$ of the carboxyl group $-\mathrm{COOH}$. 


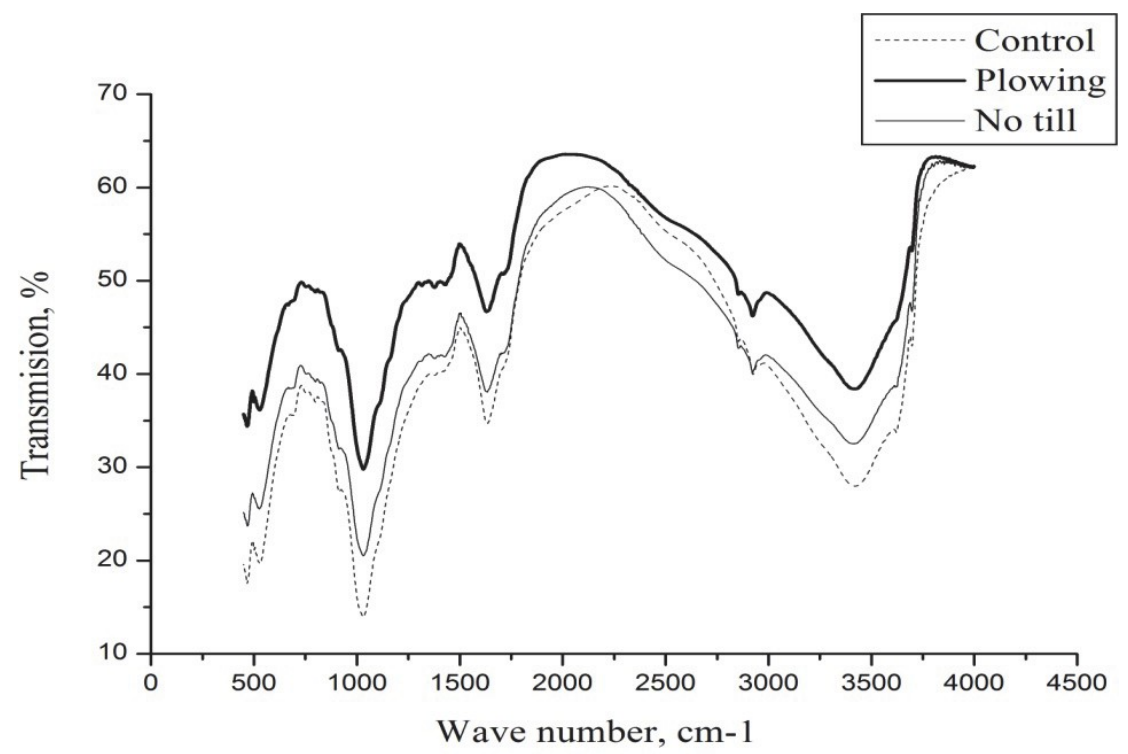

Fig. 3. FT-IR spectra of HA extracted from typical black soil under different tillage

Wide spectra range from $1800 \mathrm{~cm}^{-1}$ to $2500 \mathrm{~cm}^{-1}$ is not considered as informative, but the slope and shape (configuration) of the spectral characteristics is related to molecular weight humic acids of the examined SOM [11]. Aromatic structures are characterized by a clear band at $1625 \mathrm{~cm}^{-1}$ caused by vibrations of cyclic conjugated carbon atoms and heterocyclic compounds.

The form of IR spectra indicates the similarity of macrostructure of all investigated HA. The spectra differ in amplitudes of absorbance, slope of the line at range from $1800 \mathrm{~cm}^{-1}$ to $2500 \mathrm{~cm}^{-1}$, intensity of bands at $1440 \mathrm{~cm}^{-1}, 1375 \mathrm{~cm}^{-1}$ and $1330 \mathrm{~cm}^{-1}$, and also in amplitude of oscillations of conjugated aromatic compounds at $1625 \mathrm{~cm}^{-1}$. The tillage (plowing and No till variants) of typical black soil led to decrease in intensity of bands characteristic to aliphatic structures, compared to control soil. It is especially good expressed at the band $2800 \mathrm{~cm}-1$, due to the removal of hydrophilic aliphatic groups. For No till specimens is characteristic an increase in the amplitude of cyclic conjugated aromatic compounds due to the increased activity of polyphenoloxidase. At the range $1800 \mathrm{~cm}-1$ to $2500 \mathrm{~cm}-1$ the shape of absorbance of No till specimens is like to the same after plowing. This indicates the differences in HA molecular mass properties compared to control soil. After plowing the absorbance becomes distinctly expressed at bands $1440 \mathrm{~cm}-1,1375 \mathrm{~cm}-1$ and $1330 \mathrm{~cm}-1$, due to methyl and methoxyl groups. This is likely due to the increased activity of dehydrogenises and restructuring arrangement of functional groups.

In Fig. 4 (I) the radio frequency EPR spectrum of HA samples extracted from typical black soil under different tillage are presented. 


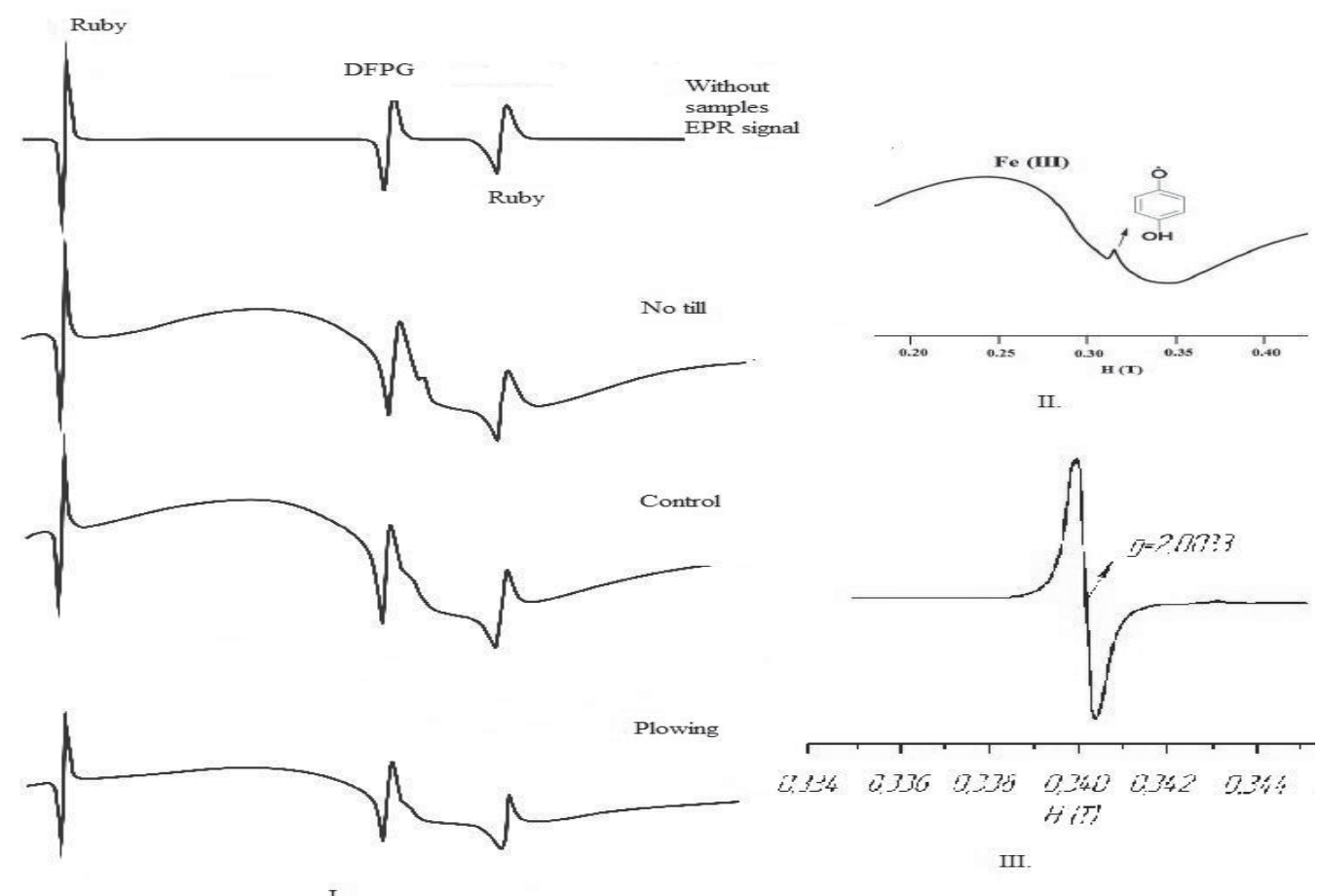

Fig. 4. EPR spectra of HA extracted from typical black soil under different tillage

EPR spectra of HA from typical black soil are characterized with g-factor value of 2.0036 . This value is close to g-factor of DPPH. EPR spectra have wide and undivided line between the points of inflection of the curve absorption of about 0.1 Tesla. This is due to the presence of chelate metal complexes with iron (III) formed as a result of rearrangement of conjugated electronic system and delocalization of electron within HA (Figure 4 II). Near the g-factor of 2.0036 is the narrow "shoulder", that corresponds to the signal of SQ radicals in the range of 0.33 to 035 Tesla (Figure 4 II), that appear as a narrow signal (Fig. 4 III).

The SQ radicals signal have the same shape but different amplitude in different samples (Fig. 5), which is associated with different concentration of cyclic aromatic structures (quinones polycondensates) in HA samples. As IR spectra show the amplitude of the signal of $S Q$ radicals decrease after plowing, which decrease of polyphenoloxidase activity. Usage of No till results in an increase in the amplitude of $S Q$ radicals, due to increased polyphenol oxidized activity. This results in an increasing of covalent bonds between structural elements and forming more hydrophobic domains in HA macrostructure.

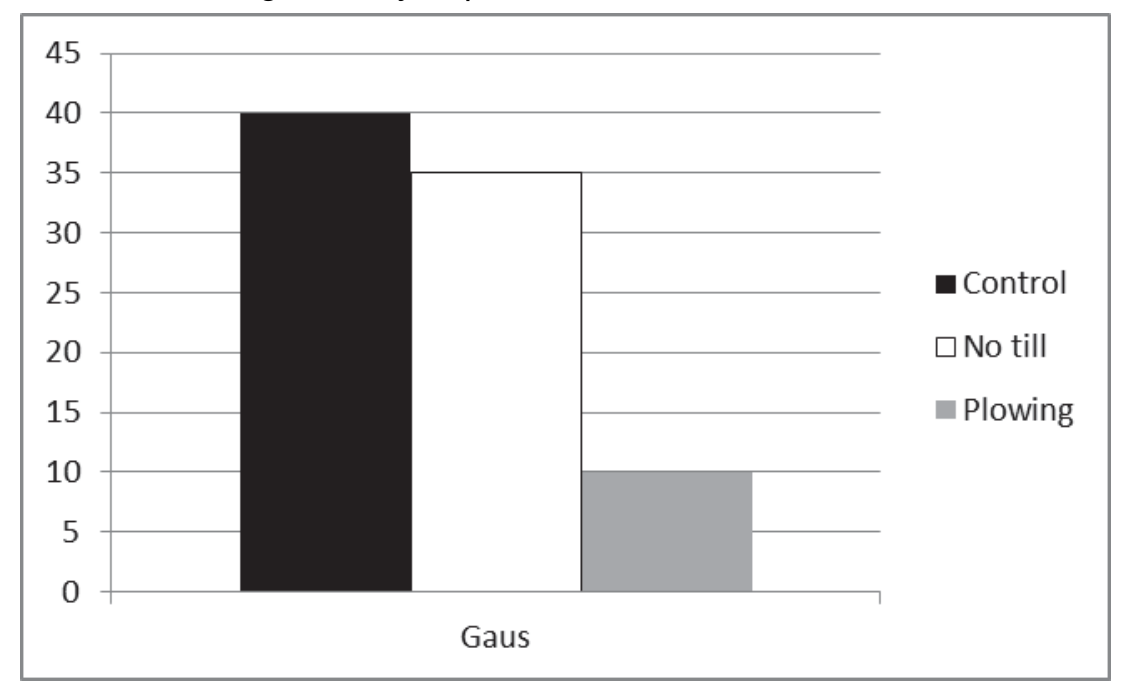

Fig. 5. The intensity of SQ radicals signal of HA extracted from typical black soil under different tillage

The profile of the molecular size distribution of HA (Fig. 6), for a given volume elution fractions is characterized by a bimodal distribution. 


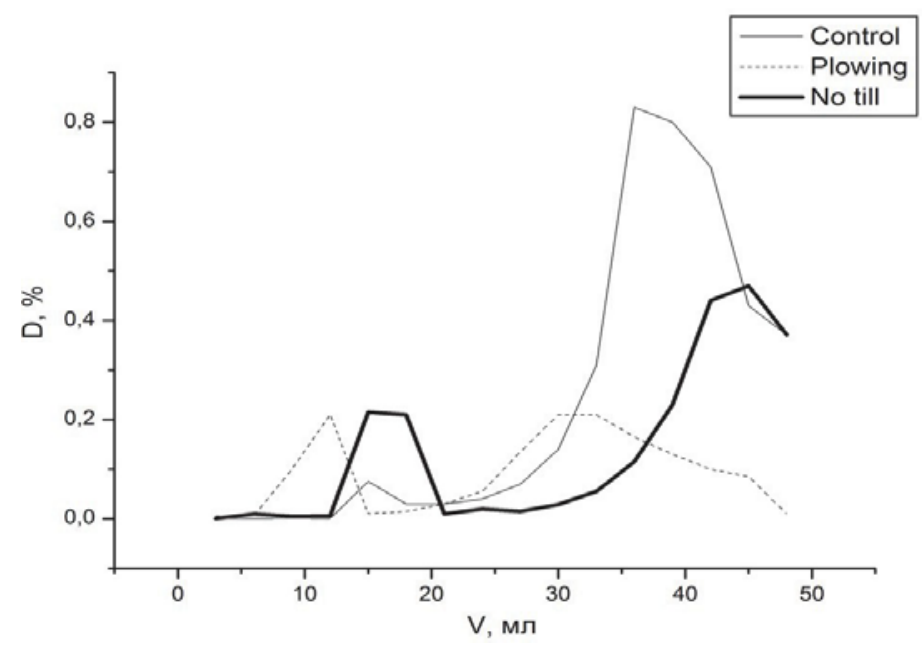

Fig. 6. Profile of molecular size distribution of $H A$ extracted from typical black soil (D, $\%$ - the intensity of the absorption wavelength at $465 \mathrm{~nm}$; $V$ - elution volume)

Large molecules that cause the first elution fractions are characterized by polar aromatic and heterocyclic structures with many oxigencontaining functional groups forming during aggregation the branched hydrophilic domains which are stabilized by $\mathrm{H}$-bounds. The second faction is associated with smaller sized molecules (and low molecular weight compounds) which are presented by nonpolar aromatic polycondensates with smaller quantity of covalent bonds. These components are associated by hydrophobic intermolecular interactions, which cause the hydrophobic nature of the faction. Profile of HA of control soil indicates the presence of more non-polar hydrophobic structures with the more intense absorption spectrum at $465 \mathrm{~nm}$ wavelength in second fraction in comparison to the first.

By plowing the elution volume changes and intensity of the absorption at wavelength $465 \mathrm{~nm}$ indicates the molecular conformation reorganized and change of amphiphiles properties of HA caused by changes in the composition of aromatic structures of HA. By plowing volume of first hydrophilic fraction increases, but the volume of hydrophobic fractions decreases, due to the reorganization of nonpolar hydrophobic low molecular weight compounds of typical black soil in hydrophilic molecular associates actively reacting with metals to form metal complexes such chelate $[4,5,10]$. Increased reactivity due hydrophilic reorganization is associated with increased activity of humus due to dehydrogenase activity blocking functional groups of aromatic structures, increasing mobility of humus and active cleavage of side aliphatic groups of HA. Therefore, the loss of carbon after plowing is associated with a decrease in low-aromatic hydrophobic domains due reorganization in hydrophilic domains and cleaved hydrophilic groups as confirmed by other studies $[1,2,6,7,10]$. After ten years of No till the elution profile of HA and increase the speed of elution indicates the increase of the quantity of hydrophobic structures, due to reversible reorganization $\mathrm{HA}$ conformation toward the formation of hydrophobic domains through increasing the number of covalent bonds due to oxidative polymerization of quinones (polycondensation) catalyzed by polyphenoloxidase.

\section{Conclusions}

1. Intensive tillage of typical black soil leads to a change of oxidoreductase activity due to increased biological mineralization of humus and to reduced system stability and decrease in resistance to decomposition of polycyclic aromatic nonpolar structures of HA. After No till s revealed an increase of polyphenoloxidase activity that leads to the increase in the quantity of covalent bonds in the conjugated polycyclic aromatic structures through oxidase polymerization (condensation), which causes an increase in non-polar hydrophobic and stable HA.

2. Dehydrogenase activity increases on plowing through "de blocking" of oxygen active functional groups due mineralization of fresh organic matter. At No till the increase of the amplitude signal of SQ radical and increase of absorption intensity of conjugated aromatic carbon structures indicate the increase of the 
quantity of hydrophobic aromatic structures. It have been shown that the use of No till leads to reversible functional reorganization of HA conformational macrostructure.

\section{References}

1. Bayer $C$. Tillage and cropping system effects on soil humic acid characteristics as determined by electron spin resonance and fluorescence spectroscopies / C. Bayer, L. Martin-Neto, J. Mielniczuk, 'et al // Geoderma.- 2002. N. 105. - P. 81-92.

2. D. S. Orlov. Humic acid soils and the general theory of humification / D. S. Orlov. - M .: MGU, 1990. $325 \mathrm{p}$

3. Piccolo A. The supramolecular structure of humic substances. A novel understanding of humus chemistry and application in Soil Science. / A. Piccolo // Advances in Agronomy. - 2002. - № 75. - P. 57133.

4. Wershaw R. L. Molecular aggregation of humic substances / R. L. Wershaw, L. Robert // Soil Science. 1999. — № 164. - P. 803-813.

5. Milanovsky E. Y. Amphihpiles component of humic substances of soil / E. Y Milanovsky // Eurasian Soil Sience. - 2000. - № 6. - P. $706-715$.

6. Tsapko Yu. L. Discussion problem of humus nature / Yu. L. Tsapko / Ukr Soil Sci. - 2015.-№ 3-4 .P. 83-89.

7. Kravchenko Y. Quality and dynamics of soil organic matter in a typical Chernozem of Ukraine under different long-term tillage systems / Y. Kravchenko, N. Rogovska, L. Petrenko // Canadian Journal of Soil Science. - 2012. - N. 92.-P. 429-438.

8. Hasiev F. H. Methods of soil enzymes / F. H. Hasiev; M.: Science, 2005.- 252 p.

9. The quality of the soil. Methods for extraction of humic substances ISO 7606:2014. - [Effective as 29.01.2014]. — K. : State Committee of Ukraine, 2014. — 8 p. — (National standard of Ukraine).

10. Popirny M. A. Effect of tillage intensity on composition, physical and chemical properties of the second fraction of humic acids extracted from typical chernozem / M. A. Popirny, O. T. Nikolov, E. V. Skrilnik // Biophysics Bulletin of Kharkov national university. - 2016. - N. 35 (10).- P. 34-45

11. Dormaar J. F. Infrared spectra of humic acids from soils formed under grass or trees / J. F. Dormaar // Geoderma.- 1967. - N. 1.- P. 37-45. 